\title{
Analysis on the Clinical Observation of Fentanyl Combined with Imidazole Valium Auxiliary Intra-spinal Canal Anesthesia Effect
}

\author{
Meijing Wang ${ }^{1, a}$, Yong Fang ${ }^{2, *}$ \\ 1 Department of Anesthesiology, The third Hospital of Jinan, Jinan, 250013,China; \\ ${ }^{2}$ Department of Pharmacy, The third Hospital of Jinan, Jinan, 250013,China \\ awangmeijing@126.com \\ *Corresponding author fangyong@126.com
}

Keywords: Imidazole Valium; Fentanyl; Spinal canal anesthesia; Analgesic

\begin{abstract}
Objective: To observe and evaluate the clinical curative effect of the Imidazole Diazepam combined with Fentanyl secondary spinal canal anesthesia sedation analgesia. Methods: We select August 2013 to October 2013 of our hospital 72 patients randomly divided into the control group and the treatment group, There are 36 patients in each group. The control group is given Imidazole intravenous drip, and the treatment group is given Imidazole Diazepam combined with Fentanyl, the intravenous drip. Results: The procedure of sedation analgesia effect comparison: the treatment group was better than control group; The intraoperative amnesia rate of the treatment group is significantly higher than control group; The intraoperative treatment group without retraction of the reaction control in pull response; Two groups of cases after sedation analgesia MAP, HR, respiratory frequency and $\mathrm{SpO} 2$ basic are in steady state, two significant differences statistically significant, $\mathrm{P}<0.05$. Conclusion: Imidazole Valium and joint Fentanyl used in spinal canal anesthesia sedation analgesia is safe and effective, the analgesic effect is good, the low incidence of adverse reactions, and is worthy of clinical popularization and application.
\end{abstract}

\section{Introduction}

The spinal canal anesthesia has incomplete anesthesia effect, clinical operations, usually with Sufentanil and Fentanyl in auxiliary induction of anesthesia. Although Fentanyl has good clinical effect, but in contrast too, for the blood flow dynamics and the influence of the cardiovascular system smaller, if used in combination with Imidazole Valium, will produce more good anterograde amnesia effect, clinical auxiliary effect is more good, has the positive medical value and is worth popularizing widely drug combinations. This article selects in August 2013 to October 2014, our hospital clinical data of 72 cases of related review analysis, study compound Fentanyl Imidazole Valium and the clinical effect of auxiliary intra-spinal canal anesthesia, now report as follows.

\section{Materials and Methods}

\section{Materials}

We select from August 2013 to October 2014, 72 cases of our hospital, were randomly divided into control group and treatment group, 36 cases in each group. Treatment group of 20 patients with male, 16 cases of patients is female, average age between $23 \sim 70$ years old, the average age of 38.6 years. The control group 21 patients with male, 15 patients with female, average age between 24 70 years old, the average age of 39.2 years. Two groups have no obvious differences in age, sex and disease, comparable.

\section{The inclusion criteria}

The control group given Imidazole stable intravenous drip, $0.03 \mathrm{mg} / \mathrm{kg}-0.04 \mathrm{mg} / \mathrm{kg}$; Treatment group given Imidazole Valium and $0.03 \mathrm{mg} / \mathrm{kg}-0.04 \mathrm{mg} / \mathrm{kg}$ joint Fentanyl, $0.15 \mu \mathrm{g} / \mathrm{kg}-0.25 \mu \mathrm{g} / \mathrm{kg}$, intravenous drip, additional half amount after $1.5 \mathrm{~h}$ to $2 \mathrm{~h}$. Homodynamic parameters in the 
preparation before and after testing, including blood pressure (MAP), heart rate (HR), respiratory frequency $(\mathrm{R})$, blood oxygen saturation (SPO2), records of force response.

\section{Statistical processing}

With mathematical statistics software SPSS19.0 for data collection and statistical analysis, and chi-square test, $\mathrm{P}<0.05$, significant difference has statistical significance.

\section{Results}

The procedure of sedation analgesia effect comparison: the treatment group was better than the control group; The intraoperative amnesia rate of the treatment group is significantly higher than control group; The intraoperative treatment group without retraction of the reaction control in pull response; Two groups of cases after sedation analgesia MAP, HR, respiratory frequency and SpO2 basic are in steady state, two significant differences statistically significant, $\mathrm{P}<0.05$.

Table 1: Two groups of cases to compare homodynamic parameters before and after treatment

\begin{tabular}{|c|c|c|c|c|c|c|}
\hline & roup & $\begin{array}{l}\text { MAP } \\
(\mathrm{mmH} \\
\mathrm{g})\end{array}$ & $\begin{array}{l}\mathrm{HR} \\
\text { (time/ } \\
\text { min) }\end{array}$ & $\begin{array}{c}\mathrm{R} \\
\text { (time/ } \\
\text { min) }\end{array}$ & $\begin{array}{c}\mathrm{SPO} 2 \\
(\%)\end{array}$ & $\begin{array}{l}\text { Pull } \\
\text { reaction }\end{array}$ \\
\hline \multirow{3}{*}{$\begin{array}{c}\text { The } \\
\text { treatme } \\
\text { nt group }\end{array}$} & $\begin{array}{l}\text { Before } \\
\text { Surgery }\end{array}$ & 79.2 & 72.2 & 14.5 & 98.4 & \multirow{3}{*}{$(-)$} \\
\hline & $\begin{array}{c}\text { Before } \\
\text { medication }\end{array}$ & 83.2 & 80.4 & 18.6 & 98.6 & \\
\hline & $\begin{array}{l}\text { In the } \\
\text { operation }\end{array}$ & 78.5 & 70.3 & 13.5 & 97.8 & \\
\hline \multirow{3}{*}{$\begin{array}{c}\text { The } \\
\text { control } \\
\text { group }\end{array}$} & $\begin{array}{l}\text { Before } \\
\text { surgery }\end{array}$ & 78.5 & 73.4 & 14.5 & 98.3 & \multirow{3}{*}{$( \pm)$} \\
\hline & $\begin{array}{c}\text { Before } \\
\text { medication }\end{array}$ & 82.3 & 81.3 & 18.7 & 98.4 & \\
\hline & $\begin{array}{l}\text { In the } \\
\text { operation }\end{array}$ & 85.3 & 84.6 & 15.4 & 98.5 & \\
\hline
\end{tabular}

Table 2: Two groups forgotten score comparison

\begin{tabular}{ccccc}
\hline Group & $\mathrm{n}$ & 2 points & 1 point & 0 point \\
\hline $\begin{array}{c}\text { The } \\
\text { treatment } \\
\text { group }\end{array}$ & 36 & $28(77.78)$ & $8(22.22)$ & $0(0)$ \\
$\begin{array}{c}\text { The control } \\
\text { group }\end{array}$ & 36 & $35(97.22)$ & $1(2.78)$ & $0(0)$ \\
\hline
\end{tabular}

\section{Discussion}

In the abdominal and lower limbs surgery, spinal canal anesthesia application is very extensive, but patients' often nervous anxiety, so need proper sedation analgesia can achieve satisfactory anesthetic effect. At present, the most commonly used auxiliary clinical anesthesia of intra-spinal canal is Imidazole plus Fentanyl, synergy, since both can increase the pain intensity, and prolonged analgesia. But the two drugs have certain respiratory inhibition. Spinal canal anesthesia in patients awake can appear all sorts of discomfort and affects the quality of anesthesia. To achieve satisfactory anesthetic effect, often need to auxiliary sedative analgesics. In recent years, Imidazole Valium compound Fentanyl has more auxiliary calm when used in local anesthesia, but improper 
dosage compatibility can cause respiratory depression and other serious complications. How to give full play to the auxiliary sedation and local anesthesia to reduce the occurrence of adverse reactions remains to be further discussed.

Before surgery, patients' often sympathetic excitement caused by the fear and anxiety, the heart rate increase fast, high blood pressure. And preoperative medication for the purpose of the inhibition of cortical, sub cortical and the limbic system, lax, emotional stability and forgetting effect, raise the pain threshold, blocking pain stimulation to the central conduction, reduce voluntary muscle activity, reduce the autonomic nerve excitability, abate the parasympathetic reflection excitability, reduce catecholamine release, antagonism of histamine, weaken the glands activities.

The surgery due to pull a appeared in the sympathetic response - the adrenal medulla and the hypothalamus - pituitary - adrenal cortex axis excitement of neuroendocrine responses. With faster heart rates, blood pressure increases, appear even obvious discomfort, particularly in had coronary artery lesions, can lead to myocardial ischemia. Imidazole Valium is a kind of anti-anxiety and calming effect good short-acting sedatives, no stimulation of vein, good anterograde amnesia, epidural anesthesia with appropriate doses of Imidazole in stability and can eliminate the patient's anxiety and fear, the patient in a state of light sleep, and have forgotten effect, small dose of Imidazole Valium and can reduce the body's stress response.

The surgery patients due to bad feelings such as tension, fear, anxiety can cause the sympathetic nervous excitement, and can make the catecholamine secretion increased significantly in the blood, which can lead to heart rate increase fast, blood pressure, and increase the patient's adverse emotional reaction, causing a vicious cycle. At present, Fentanyl combined Imidazole Valium and auxiliary anesthesia has been relatively matures, Imidazole Valium sedative effect is good, can obviously relieve tension, anxiety patients, and can produce effect. Fentanyl for potent analgesic can strengthen the anesthetic.

In recent years, Imidazole Valium compound Fentanyl is widely used in clinical anesthesia, Imidazole were reported in the literature as a stable compound Fentanyl fast as an aid in the medicine has the effect of local anesthesia, calm, and the advantages of the combined effect is strong, short patients awake time. But, if use improper dosage, combined the two drugs used for auxiliary calm when the local anesthetic, could significantly inhibit the patient's respiratory function, adverse complications. Application, how to match the two drugs both can have better sedation and analgesia effect, and try to avoid its side effects, therefore, we turn to the two drugs in the clinical dosage of common reference and related research literature, designed the compatibility of different doses of Imidazole Valium and joint Fentanyl used for auxiliary local anesthesia, through record calming effect and side effects, compared to determine the best compatibility of the two drugs, the local anesthetic for application of the two drugs in auxiliary to provide reference and basis. In local anesthesia to attain ideal depth of sedation, patients should be able to keep patients during anesthesia can awaken in shallow Morpheus condition at any time, eliminate the anxiety and tension, intraoperative patients can keep respiratory tract unobstructed and spontaneous breathing was maintained, and have anterograde amnesia.

The analgesic effect can reduce the patients viscera pull when feeling unwell. Both share can produce synergies, inhibit respiration, so should be paid attention to when using the in reduce the dosage. Fentanyl is Fentanyl derivatives, its analgesic effect is higher than that of Fentanyl, use more secure, pain intensity is $5 \sim 10$ times that of Fentanyl and Fentanyl easier through the blood brain barrier, lasts longer, the degree of respiratory depression and Fentanyl. This group of patients did not show obvious respiratory depression, may be related to the operation process, the dosage are less relevant. Patients with spinal canal anesthesia for incomplete anesthesia, intraoperative can appear negative emotions, such as tension, anxiety, irritability, and patients with visceral perception pull can aggravate the patient's discomfort and pain experience, sedative and analgesic drugs commonly used for auxiliary anesthesia operation, the Fentanyl combined Imidazole is settled in clinical anesthesia effect have been sure of. Fentanyl is Fentanyl derivatives, its has quick effect, 
analgesic effect is good and stable efficacy, etc, our hospital in recent years, using Fentanyl combined Imidazole Valium and auxiliary intra-spinal canal anesthesia, obtaining good results.

Existed during anesthesia and surgery in patients with different degree of fear and anxiety psychology, the psychological causes parasympathetic nerve reflex, make patients slow heart rate, blood pressure drops, nausea and vomiting; Adrenaline, catecholamine secretion to increase, the heart rate, blood pressure, blood glucose, etc.; Long time to maintain the operation position, also brings to the patients with lower limb numbness, dry mouth, agitated discomfort; Sane, intraoperative anesthesia patients exist clear consciousness of fear and anxiety in the process of operation, leading to emotional tension and fear, to bring certain psychological trauma patients, this kind of influence the postoperative recovery of patients with trauma, even for a long period of time after surgery has certain mental disorders, bring lasting damage. Narcotic drugs properly, make good preoperative sedation, ensure the memory operation.

The Imidazole Valium and can be used for intravenous drip, and can be used for muscle injection, and drug dose and effect is positively correlated, drug dosage increased, calm, anticonvulsants, relaxation, hypnotic amnesia and line also increases. Therefore intra-spinal canal anesthesia used Imidazole can achieve the analgesic effect of moderate, good stability, to maintain homodynamic stability. Keep good consciousness, patients with intraoperative surgery can adapt to the performer, and can make patients postoperative intact. Imidazole diazepam has anti-anxiety, composed, forgetfulness, anticonvulsants, muscle relaxant effects of short-acting benzodiazepines drugs that are widely used in the clinical; research has proved that the preoperative use of Imidazole Valium can produce good anterograde amnesia. In the general anesthesia, appropriate sedation is very necessary to patients, satisfaction of calm can eliminate the patient's anxiety and fear of anesthesia surgery; Operations in the process of pain, discomfort, pull and all kinds of bad stimulation on patients prognosis and psychological impact, as a result, many patients require surgery done in sleep state.

Fentanyl is opioid agonist, is a potent narcotic analgesics, strengthening the analgesic effect of local anesthesia, reduce all kinds of discomfort to patients and improve patient comfort and the effect of anesthesia. The spinal canal anesthesia used Imidazole Valium compound Fentanyl, combined use of two drug has synergistic anesthesia effect, can reduce the dose of the medicine. Imidazole Valium compound Fentanyl anesthesia work fast, calm and combined effect is strong, fast recovery, drug resistance. Fentanyl and Imidazole Valium share lower dose of the two drugs, the dosage of lower also reduces the drug adverse reaction.

In recent years, Imidazole Valium compound Fentanyl as anesthetic sedative drug combinations of intra-spinal canal is widely used in clinic, many literatures have reported Imidazole diazepam combined Fentanyl as adjuvant of vertebral canal anesthesia has quick effect, calm and strong effect, patients recover quickly, the characteristics of the drug can be antagonism. Imidazole Valium is a potent sedative medicine, can alleviate the patient's anxiety and tension, Central Line muscle relaxation and amnesia effect, but mild control breathing, inhibition degree related to the dose and drug injection rate. Fentanyl is a potent analgesic, can strengthen the parts of anesthesia analgesic action, relieve the discomfort of surgery in patients with abdominal viscera pull produce, and greatly improve the effect of anesthesia. Fentanyl and Imidazole Valium share can greatly reduce the dosage of the drug, reduce drug adverse reactions, but the two together also exists synergistic effect on respiratory function, inhibition of should pay attention to the breath, the analgesic effect of Fentanyl was stronger than Fentanyl, is currently the strongest analgesic action of narcotic analgesics, analgesic effect of Fentanyl in 5 to 10 times, with a range of security, which is about 100 times that of Fentanyl, lasts longer, about 2 times of Fentanyl, to the degree of respiratory depression and equivalent dose of Fentanyl is similar. Because of Fentanyl suppression time is short of breath after the operation and the possibility of morphine mechanism occurred in small, so after surgery, the patient's respiratory management compared with Fentanyl simple security improved.

Group min $75 \% 3$ patients appeared different degree of the medicine calm, calm peak for $3 \sim 15$ min after medication, there will be a short sleep more than $50 \%$ of the patients, but easy to wake up, only $7 \%$ of the patients with strong stimulation to wake up. Of Fentanyl analgesic action is 
beneficial to relieve the intense discomfort, such as skin and to probe the early surgery if used alone this dose Fentanyl almost no sleep. 30 min after more than half of patients fully awake, reply freely. But there is still obvious sedative effect, no anxiety agitation, and combined effect is strong. Because pure mostly patients under local anesthesia in the waking state, how to make the patients avoid bad memory in surgery is an important task in clinical anesthesia work. With Imidazole Valium has strong characteristics of the role of anterograde amnesia, it can avoid to surgery in patients with adverse stimuli in memory, in the process of elimination of postoperative mental trauma. Four groups of patients in this study can maintain a higher rate of anterograde amnesia, same as the other related results reported in literatures. In addition, Fentanyl and Imidazole Valium of respiratory inhibition and individual susceptibility to drug related, reflected in our experiments, the dosage of the smallest group A 5\% incidence of respiratory depression. This study adopted by the compatibility combination dose not lead to obvious inhibition, is safe and effective.

But Imidazole diazepam has no analgesic action, in order to eliminate local anesthesia patient discomfort and pain in the anesthesia and surgery is usually a combination of analgesic drugs. Fentanyl is a potent opioid, work fast, effect time is shorter, used for the local anesthetic sedative can strengthen the effect of local anesthetics and sedatives sedative effects, and can reduce the dosage of sedatives. In recent years, Imidazole valium plus Fentanyl used widely in clinic, many literatures have reported Imidazole valium as a local anesthetic sedative, plus Fentanyl has quick effect, calm and strong effect, patients recover quickly, the characteristics of the drug can be antagonism. However, Imidazole valium + Fentanyl combined local anesthetic used to calm, to the patient's respiratory function inhibition of obvious synergy, if it is used undeserved also can cause adverse consequences.

\section{Conclusion}

This research shows that the Imidazole Valium and Fentanyl compound curative effect is applied in the spinal canal anesthesia, and the sedation effect is remarkable, and it has good effect of forgetting. But Imidazole Valium and Fentanyl are to inhibit the action of the respiratory function; two drug combinations also have synergy to inhibition of respiratory function. And Imidazole Valium and Fentanyl compound anesthesia effects on heart rate and blood pressure also have synergy; the blood pressure particularly evident in the spinal canal anesthesia is dropped. Therefore Imidazole Valium and Fentanyl compound intra-spinal canal anesthesia to patients with appropriate rehydration, by supplementing blood volume in order to assure the normal blood pressure. The low incidence of adverse reactions, and is worthy of clinical popularization and application.

\section{Acknowledgement}

This research was financially supported by the National Science Foundation.

\section{References}

[1] Conti G, Arcangeli A, Antonelli M, et al. Sedation with sufentanil in patients receiving pressure support ventilation has no effects on respiration: a pilot study. Canadian Journal of Anaesthesia. 2012.

[2] Mora CT, Torjman M, White PF. Sedative and ventilatory effects of midazolam infusion: Effect of flumazenil reversal. Canadian Journal of Anaesthesia. 2010.

[3] ADSA.ADSA guidelines for intraoperative monitoring of patients undergoing conscious sedation. ADSA Newsletter. 2012.

[4] Scamman FL, Klein SL, Choi WW. Conscious sedation for procedures under local or topical anesthesia. Annals of Otology Rhinology Laryngology. 2013.

[5] Chernik DA, Gillings D,Laine H, et al.Validity and reliability of the Observer's Assessment of Alertness/Sedation Scale: study with intravenous midazolam. Journal of Clinical Psychopharmacology. 2012. 
[6] Nishiyama T, Yokoyama T, Hanaoka K. Sedation guidelines for midazolam infusion during combined spinal and epidural anesthesia. Joumal of Clinical Anesthesia. 2012.

[7] White PF, Negus JB. Sedative infusions during local and regional anesthesia: a comparison of midazolam and propofol. Journal of Clinical Anesthesia. 2012.

[8] Green SM, Hummel CB, Wittlake WA, et al. What is the optimal dose of intramuscular ketamine for pediatric sedation. Academic Emergency Medicine . 2012.

[9] McGlone RG, Howes MC, Joshi M. The Lancaster experience of 2.0 to $2.5 \mathrm{mg} / \mathrm{kg}$ intramuscular ketamine for paediatric sedation: 501 cases and analysis. Emergency Medicine Journal. 2011.

[10] Heinz P, Geelhoed GC, Wee C, Pascoe EM.Is atropine needed with ketamine sedation? A prospective, randomised, double blind study. Emergency Medicine Journal. 2010. 